GANIT J. Bangladesh Math. Soc. (ISSN 1606-3694) 34 (2014) 63-73

\title{
EFFECTS OF CONDUCTION VARIATION ON MHD NATURAL CONVECTION FLOW ALONG A VERTICAL FLAT PLATE
}

\author{
A.K.M. Safiqul Islam ${ }^{1}$, M. A. Alim ${ }^{2}$, Md. Rezaul Karim ${ }^{3}$ and ATM. M. Rahman ${ }^{4}$ \\ ${ }^{1}$ Department of Mathematics, Gowripur Govt. College, Mymensingh, Bangladesh. \\ ${ }^{2}$ Department of Mathematics, Bangladesh University of Engineering and Technology, \\ Dhaka-1000, Bangladesh. \\ ${ }^{3}$ Department of Mathematics, Jagannath University, Dhaka-1100, Bangladesh \\ ${ }^{4}$ Department of Computer Science and Engineering, Dhaka International University, Banani, \\ Dhaka-1213, Bangladesh. \\ Email: safiqis@yahoo.com, maalim@math.buet.ac.bd, rrzauljnu@gmail.com, \\ mrrahmandiu@yahoo.com
}

Received 14.09.2014

Accepted 07.12.2014

\begin{abstract}
This paper reports free convection flow along a vertical flat plate with conduction variation on magneto hydrodynamic (MHD) effects. The governing equations with associated boundary conditions reduce to local non-similarity boundary layer equations for this phenomenon are converted to dimensionless forms using a suitable transformation. The transformed non-linear equations are then solved using the implicit finite difference method together with Keller-box technique. Numerical results of the velocity and temperature profiles, skin friction and surface temperature profiles for different values of the magnetic parameter, the Prandtl number and the conduction variation parameters are presented graphically. Detailed discussion is given for the effect of the aforementioned parameters.
\end{abstract}

Keywords: Flat plate, Electrically conducting fluid, Natural convection, Conduction variation

\section{Introduction}

Electrically conducting fluid flow in presence of MHD and heat conduction problems are important from the technical point of view and such types of problems have received much attention by many researchers.

Model studies of the free convection flows have earned reputations because of their applications in geophysical, geothermal and nuclear engineering problems. Pozzi and Lupo [1] investigated the coupling of conduction with laminar convection along a flat plate. Pop et al. [2] investigated the conjugate mixed convection on a vertical surface in porous medium. Gebhart [3] investigated the effect of dissipation natural convection. Takhar and Soundalgekar [4] studied the dissipation effects on MHD free convection flow past a semi-infinite vertical plate. Khan [5] investigated the conjugate effect of conduction and convection with natural convection flow from a vertical flat plate and in an inclined square cavity. Mamun [6] studied the effects of conduction and convection on magnetohydrodynamic flow with and without viscous dissipation from a vertical flat plate. Hossain [7] analyzed the viscous and Joule heating effects on MHD free convection 
flow with variable plate temperature. Rahman et al. [8] investigated the effects of temperature dependent thermal conductivity on MHD free convection flow along a vertical flat plate with heat conduction. Rahman and Alim [9] analyzed numerical study of MHD free convective heat transfer flow along a vertical flat plate with temperature dependent thermal conductivity. Alim et al. [10] analyzed the Combined effect of viscous dissipation \& joule heating on the coupling of conduction \& free convection along a vertical flat plate. Alim et al. [11] investigated Joule heating effect on the coupling of conduction with MHD free convection flow from a vertical flat plate. Chowdhury and Islam [16] analyzed MHD Free Convection Flow of Visco-elastic Fluid past an Infinite Porous Plate. Alam et al. [17] studied viscous dissipation effects on MHD natural convection flow over a sphere in the presence of heat generation. Islam et al. [18] assessed Effects of thermal conductivity of fluid on free convection flow along a vertical flat plate with transverse conduction.

The present study is to incorporate the idea of the effects of conduction variation on MHD free convection boundary layer flow along a vertical flat plate.

\section{Mathematical formulation of the problem}

We consider a steady two-dimensional laminar natural convection flow of an electrically conducting, viscous and incompressible fluid along a vertical flat plate of length $l$ and thickness $b$ (Figure-1). It is assumed that the temperature at the outer surface of the plate is maintained at a constant temperature $T_{b}$, where $T_{b}>T_{\infty}$, the ambient temperature of the fluid. In this work $\bar{y}$-axis i.e. normal direction to the surface and $\bar{x}$-axis is taken along the flat plate. The coordinate system and the configuration are shown in Fig.-1.

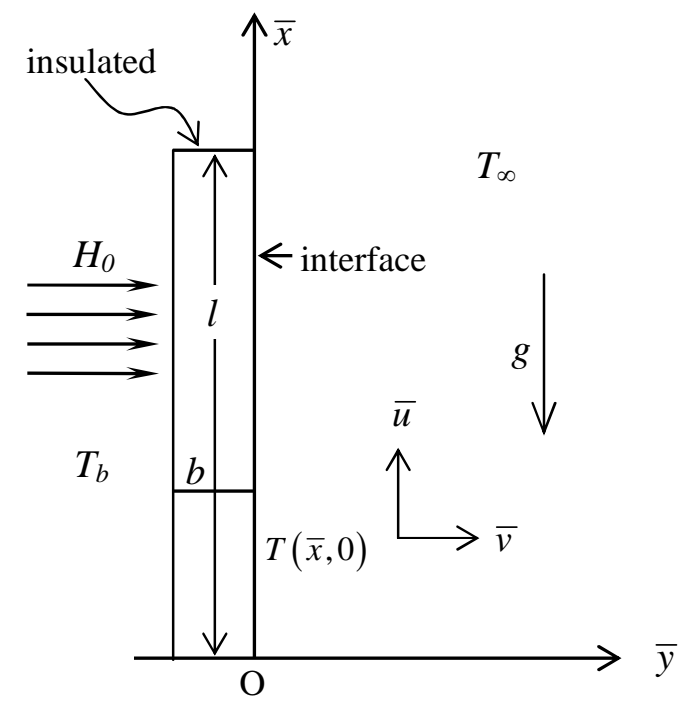

Fig. 1: Physical model and coordinate system

The governing equations of such laminar flow with MHD and also thermal conductivity variation along a vertical flat plate under the Boussinesq approximations 
$\rho=\rho_{\infty}\left[1-\beta\left(T_{b}-T_{\infty}\right)\right]$, where $\rho_{\infty}$ and $T_{\infty}$ are the density and temperature respectively outside the boundary layer. For the present problem for continuity, momentum and energy equations take the following forms

$$
\begin{aligned}
& \frac{\partial \bar{u}}{\partial \bar{x}}+\frac{\partial \bar{v}}{\partial \bar{y}}=0 \\
& \bar{u} \frac{\partial \bar{u}}{\partial \bar{x}}+\bar{v} \frac{\partial \bar{v}}{\partial \bar{y}^{2}}=v \frac{\partial^{2} \bar{u}}{\partial \bar{y}^{2}}+g \beta\left(T_{f}-T_{\infty}\right)-\frac{\sigma H_{0}^{2} \bar{u}}{\rho} \\
& \bar{u} \frac{\partial T_{f}}{\partial \bar{x}}+\bar{v} \frac{\partial T_{f}}{\partial \bar{y}}=\frac{1}{p C_{p}} \frac{\partial}{\partial \bar{y}}\left(\kappa_{f} \frac{\partial T_{f}}{\partial \bar{y}}\right)
\end{aligned}
$$

Here $\beta$ is coefficient of volume expansion. The temperature dependent thermal conductivity, which is used by Rahman [8] as follows $\kappa_{f}=\kappa_{\infty}\left[1+\delta\left(T_{f}-T_{\infty}\right)\right]$

Where $\kappa_{\infty}$ is the thermal conductivity of the ambient fluid and $\delta$ is a constant, defined as $\delta=\frac{1}{\kappa_{f}}\left(\frac{\partial \kappa}{\partial T}\right)_{f}$. The appropriate boundary condition to be satisfied by the above equations are

$$
\begin{aligned}
& \left.\begin{array}{l}
\bar{u}=0, \bar{v}=0 \\
T_{f}=T(\bar{x}, 0), \frac{\partial T_{f}}{\partial y}=\frac{\kappa_{s}}{b \kappa_{f}}\left(T_{f}-T_{b}\right)
\end{array}\right\} \text { an } \bar{y}=0, \bar{x}>0 \\
& \bar{u} \rightarrow 0, T_{f} \rightarrow T_{\infty} \text { as } \bar{y} \rightarrow \infty, \bar{x}>0
\end{aligned}
$$

The non-dimensional governing equations and boundary conditions can be obtained from equations (1) - (3) using the following dimensionless quantities

$$
\begin{aligned}
& x=\frac{\bar{x}}{l}, y=\frac{\bar{y}}{l} G r^{\frac{1}{4}}, u=\frac{\bar{u} l}{v} G r^{-\frac{1}{2}}, v=\frac{\bar{v} l}{v} G r^{-\frac{1}{4}} \\
& \theta=\frac{T_{f}-T_{\infty}}{T_{b}-T_{\infty}}, G r=\frac{g \beta l^{3}\left(T_{b}-T_{\infty}\right)}{v^{2}}
\end{aligned}
$$

where $l$ is the length of the plate, $G r$ is the Grashof number, $\theta$ is the dimensionless temperature.

Now from equations (1)-(3), we get using the following dimensionless equations

$$
\begin{aligned}
& \frac{\partial u}{\partial x}+\frac{\partial v}{\partial y}=0 \\
& u \frac{\partial u}{\partial x}+v \frac{\partial v}{\partial y}+M u=\frac{\partial^{2} u}{\partial y^{2}}+\theta
\end{aligned}
$$




$$
u \frac{\partial \theta}{\partial x}+v \frac{\partial \theta}{\partial y}=\frac{1}{\operatorname{Pr}}(1+\gamma \theta) \frac{\partial^{2} \theta}{\partial y^{2}}+\frac{\gamma}{\operatorname{Pr}}\left(\frac{\partial \theta}{\partial y}\right)^{2}
$$

where $\operatorname{Pr}=\frac{\mu C_{p}}{\kappa_{\infty}}$ is the Prandtl number, $\gamma=\delta\left(T_{b}-T_{\infty}\right)$ is the non-dimensional thermal conductivity variation parameter and $M=\frac{\sigma H_{0}^{2} l^{2}}{\mu G r^{1 / 2}}$ is the dimensionless magnetic parameter. The corresponding boundary conditions (5) then take the following form

$$
\begin{aligned}
& u=0, v=0, \theta-1=(1+\gamma \theta) P \frac{\partial \theta}{\partial y} \text { on } y=0, x>0 \\
& u \rightarrow 0, \theta \rightarrow 0 \text { as } y \rightarrow \infty, x>0
\end{aligned}
$$

here $P=\left(\frac{\kappa_{\infty} b}{\kappa_{s} l}\right) G r^{\frac{1}{4}}$ is the conduction variation parameter. The described problem is governed by the conduction variation parameter $P$.

To solve the equations (8) and (9) subject to the boundary conditions (10) the following transformations are proposed by Merkin \& Pop [12]

$$
\begin{aligned}
& \psi=x^{\frac{4}{5}}(1+x)^{-\frac{1}{20}} f(x, \eta) \\
& \eta=y x^{-\frac{1}{5}}(1+x)^{-\frac{1}{20}} \\
& \theta=x^{\frac{1}{5}}(1+x)^{-\frac{1}{5}} h(x, \eta)
\end{aligned}
$$

here $\eta$ is the similarity variable and $\psi$ is the non-dimensional stream function which satisfies the continuity equation and is related to the velocity components in the usual way as

$$
u=\frac{\partial \psi}{\partial y} \text { and } v=-\frac{\partial \psi}{\partial x}
$$

Moreover, $h(x, \eta)$ represents the non-dimensional temperature. The momentum and energy equations are transformed for the new co-ordinate system. At first, the velocity components are expressed in terms of the new variables for this transformation. Thus the following equations

$$
f^{\prime \prime \prime}+\frac{16+15 x}{20(1+x)} f f^{\prime \prime}-\frac{6+5 x}{10(1+x)} f^{\prime 2}-M x^{\frac{2}{5}}(1+x)^{\frac{1}{10}} f^{\prime}+h=x\left(f^{\prime} \frac{\partial f^{\prime}}{\partial x}-f^{\prime \prime} \frac{\partial f}{\partial x}\right)
$$




$$
\begin{aligned}
& \frac{1}{\operatorname{Pr}} h^{\prime \prime}+\frac{\gamma}{\operatorname{Pr}}\left(\frac{x}{1+x}\right)^{\frac{1}{5}} h h^{\prime \prime}+\frac{\gamma}{\operatorname{Pr}}\left(\frac{x}{1+x}\right)^{\frac{1}{5}} h^{\prime 2}+\frac{16+15 x}{20(1+x)} f h^{\prime} \\
& -\frac{1}{5(1+x)} f^{\prime} h=x\left(f^{\prime} \frac{\partial h}{\partial x}-h^{\prime} \frac{\partial f}{\partial x}\right)
\end{aligned}
$$

where prime denotes partial differentiation with respect to $\eta$. The boundary conditions as mentioned in equation (10) then take the following form

$$
\begin{aligned}
& f(x, 0)=f^{\prime}(x, 0)=0 \\
& h(x, 0)=\frac{x^{\frac{1}{5}}(1+x)^{-\frac{1}{5}} h(x, 0)-1}{(1+x)^{-\frac{1}{4}}+\gamma x^{\frac{1}{5}}(1+x)^{-\frac{9}{20}} h(x, 0)} \\
& f^{\prime}(x, \infty) \rightarrow 0, h(x, \infty) \rightarrow 0
\end{aligned}
$$

From the process of numerical computation, in practical point of view, it is important to calculate the values of the surface shear stress in terms of the skin friction coefficient. This can be written in the non-dimensional form as Molla et al. [13]

$$
C_{f}=\frac{G r^{-\frac{3}{4}} l^{2}}{\mu v} \tau_{w}
$$

where $\tau_{w}\left[=\mu(\partial \bar{u} / \partial \bar{y})_{\bar{y}=0}\right]$ is the shearing stress. Using the new variables described in (6), the local skin friction coefficient can be written as

$$
C_{f x}=x^{\frac{2}{5}}(1+x)^{-\frac{3}{20}} f^{\prime \prime}(x, 0)
$$

In practical point of view, it is important to calculate the values of the surface temperature. The numerical values of the surface temperature are obtained from the relation. This can written in the non-dimensional form as

$$
\theta(x, 0)=x^{\frac{1}{5}}(1+x)^{-\frac{1}{5}} h(x, 0)
$$

\section{Numerical Solution}

This paper investigates the effect of conduction variation on electrically conducting fluid in natural convection flow along a vertical flat plate with MHD. The set of equations (12) and (13) together with the boundary conditions (14) are solved by applying implicit finite difference method with Keller-box elimination scheme [14], which is well documented by Cebeci and Bradshaw [15] and widely used by Keller and Cebeci and Hossain. 


\section{Results and discussion}

The main objective of the present study is to analyze the effect of conduction variation due to temperature on free convective flow along a vertical flat plate in presence of strong magnetic field. In this simulation the values of the Prandtl number $\mathrm{Pr}$ are considered to be $0.73,1.00,1.50,2.00$ and 2.50 that corresponds to hydrogen, steam, water, methyl chloride and sulfur dioxide respectively.

The velocity and the temperature profiles obtained from the solutions of equations (12) and (13) are depicted in Figures 2 to 4. Also the local skin friction and the surface temperature obtained from the solutions of equations (16) and (17) are depicted in Figures 5 to 7. Numerical computation are carried out for a range of magnetic parameter $M=$ $0.02,0.20,0.40,0.60,0.80$ and conduction variation parameter $P=0.10,0.16,0.22,0.28$, 0.35 .

The effect of magnetic parameter $M$ on the velocity and temperature profiles against $\eta$ within the boundary layer with $P r=1.73$ and $P=0.10$ are shown in Fig. 2(a) and 2(b), respectively. It is seen from Fig. 2(a) that the velocity decreases within the boundary layer with the increasing values of $M$. On the other hand temperature increases within the boundary layer with the increasing values of $M$. It means that the velocity boundary layer and the thermal boundary layer thickness expand inversely for large values of $M$.

Fig. 3(a) and 3(b) illustrate the velocity and temperature profiles against $\eta$ for different values of Prandtl number $\operatorname{Pr}$ with $M=0.10$ and $P=0.10$. From Fig. 3(a), it can be observed that the velocity decreases as well as its position moves toward the interface with the increasing $P r$. From Fig. 3(b), it is seen that the temperature profiles shift downward with the increasing values of $P r$. It means that the velocity boundary layer and the thermal boundary layer thickness expand clearly in same way for large values of $P r$.

In Figures 4(a) and 4(b) describe the velocity and temperature profiles against $\eta$ for different values of conduction variation parameter $P$ with $M=0.10$ and $\operatorname{Pr}=1.73$. From Fig. 4(a), it can be observed that the velocity decreases as well as its position moves outward the interface with the increasing values of $P$. From Fig. 4(b), it is seen that the temperature profiles also the same as increasing within the boundary layer. It means that the velocity boundary layer and the thermal boundary layer thickness decrease for large values of $P$ from 0.10 to 0.35 . There is an inverse trend is found in velocity boundary layer.

Figure 5(a) and Figure 5(b) illustrate the effect of the magnetic parameter on the local skin friction coefficient $C_{f x}$ and surface temperature distribution $\theta(x, 0)$ against $x$ with $P r$ $=1.73$ and $P=0.10$. It is seen from Figure 5(a) that the skin friction increases monotonically along the upward direction of the plate for a particular value of $M$. It is also seen that the local skin friction coefficient decreases for the increasing values of $M$. From Figure 5(b), it can be seen that the surface temperature increases due to the increasing value along the positive $x$ direction for a particular $M$. It is also observed that the surface temperature decreases for increasing values of $M$. This means that there is an inverse case exists between skin friction and surface temperature which is remarkable.

Figure 6(a) and Figure 6(b) deal with the effect of Prandtl number Pr on the skin friction and surface temperature against $x$ with $M=0.10$ and $P=0.10$. It can be observed from 
Figure 6(a) that the skin friction decreases monotonically for a particular value of $P r$. It can also be noted that the skin friction decreases for the increasing values of $P r$. From Figure 6(b), it can be seen that the surface temperature increases due to the increasing along the positive $x$ direction for a particular value of $P r$. This means that there is an inverse case exists between skin friction and surface temperature which is mentionable.

The variation of the skin friction and surface temperature for different values of $P$ with $M$ $=0.10$ and $P r=1.73$ at different positions are illustrated in Figures 7(a) and 7(b), respectively. Skin friction decreases for increasing values of $P$. It can also be noted from Figure 7(a) that the skin friction increases monotonically for a particular value of $P$. Again Figure 7(b) shows that the surface temperature increases for increasing values of $P$. We observe from the profiles of skin friction and surface temperature that they are opposite.
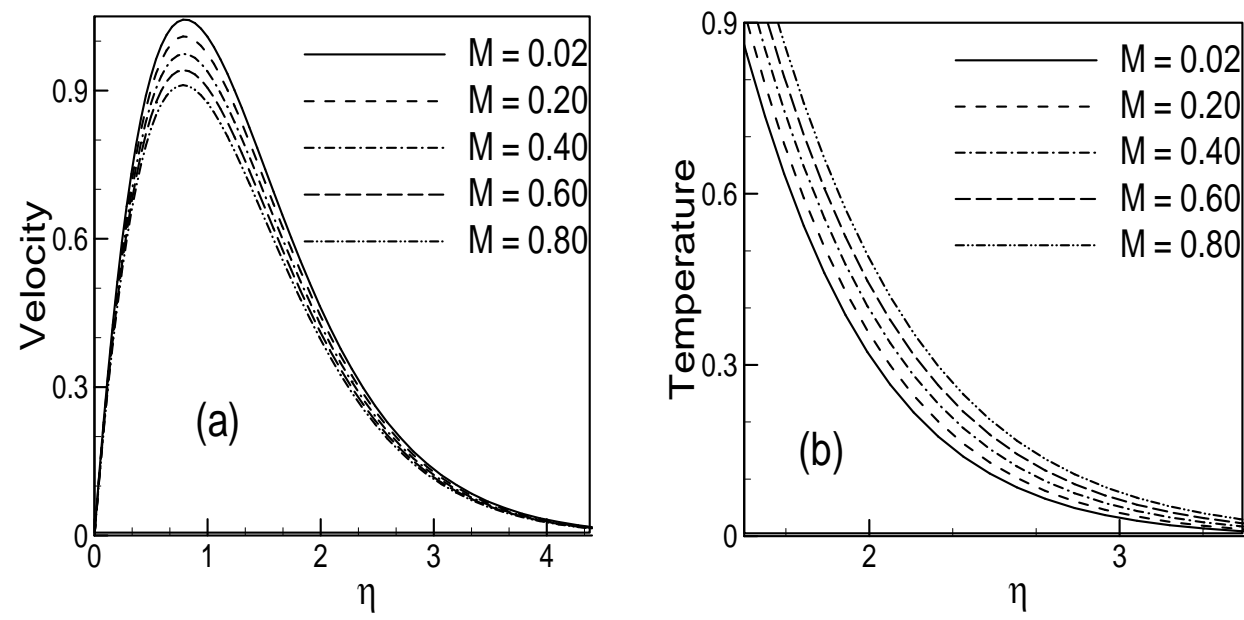

Fig. 2(a) Velocity and (b) Temperature profiles against $\eta$ for different values of $M$ with $P r=1.73$ and $P=0.10$.
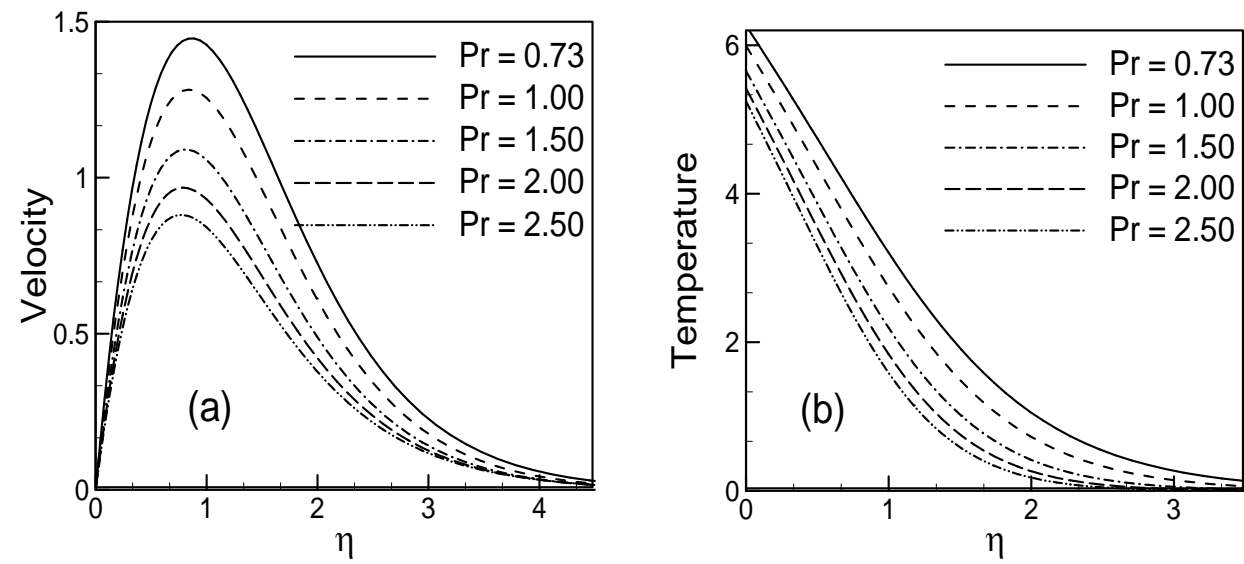

Fig. 3(a) Velocity and (b) Temperature profiles against $\eta$ for different values of $\operatorname{Pr}$ with $M=0.10$ and $P=0.10$. 

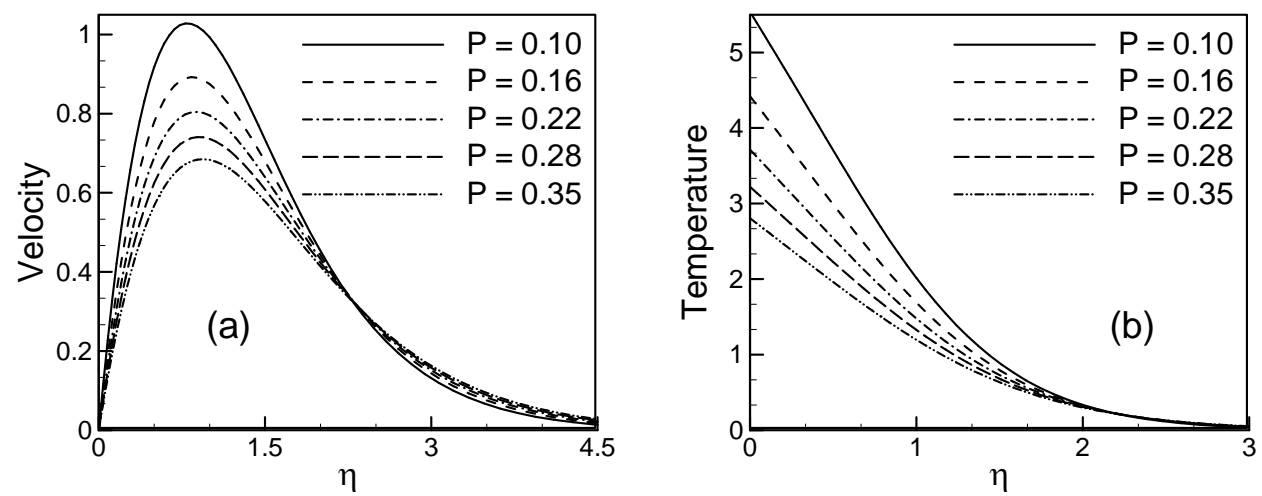

Fig. 4(a) Velocity and (b) Temperature profiles against $\eta$ for different values of $P$ with $M=0.10$ and $\operatorname{Pr}=1.73$.
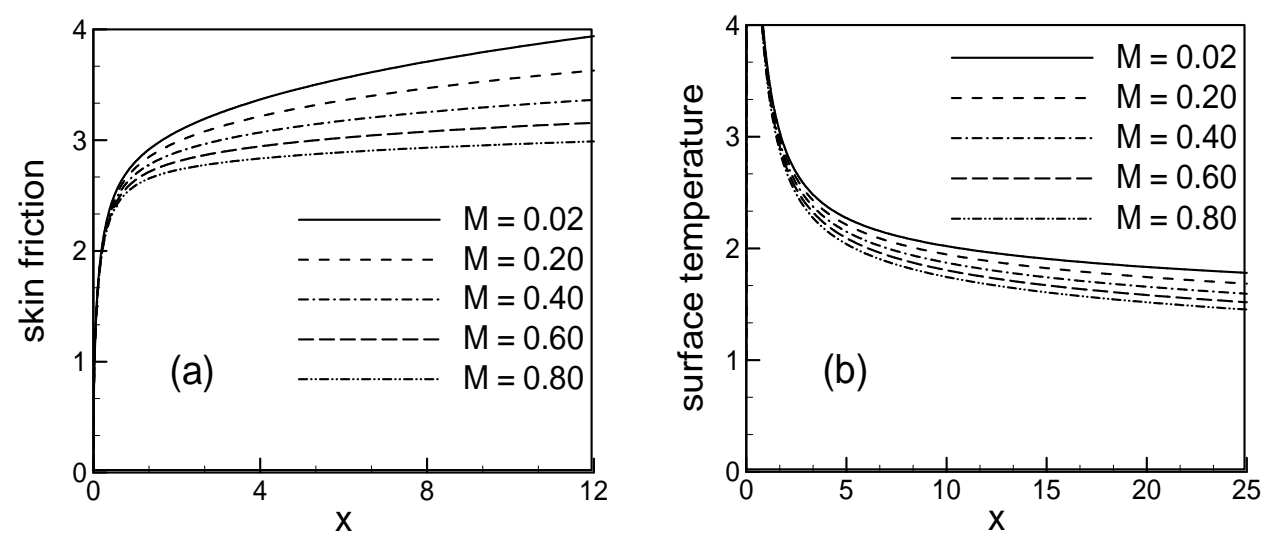

Fig. 5(a) Local skin friction coefficient and (b) Surface temperature distribution against $x$ for different values of $M$ with $\operatorname{Pr}=1.73$ and $P=0.10$.
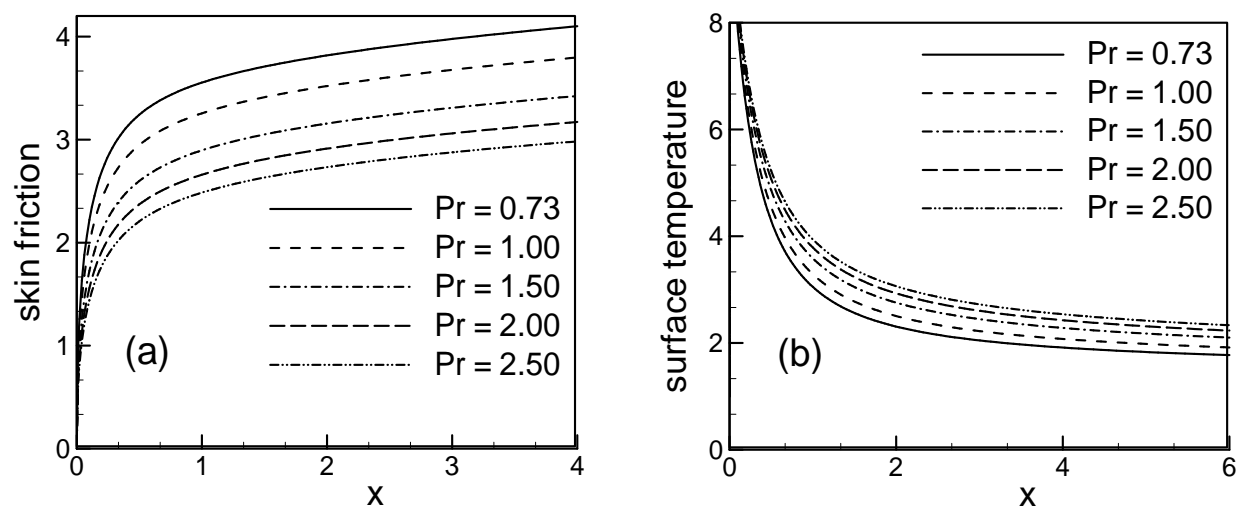

Fig. 6(a) Skin friction coefficient and (b) Surface temperature against $x$ for different values of $\operatorname{Pr}$ with $M=$ 0.10 and $P=0.10$ 

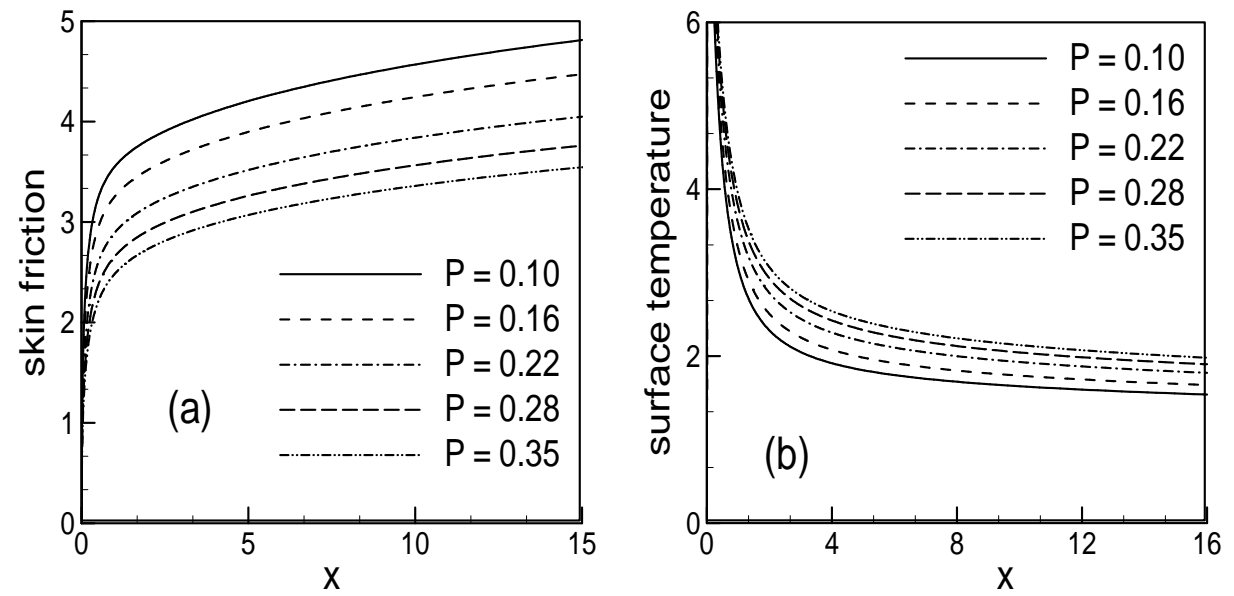

Fig. 7(a) Skin friction and (b) Surface temperature against $x$ for different values of $P$ with $M=0.10$ and $P r=1.73$.

\section{Comparison of the results}

Table 1 and Table 2 depict the comparisons of the present numerical results of the skin friction $C_{f x}$ and the surface temperature $\theta(x, 0)$ with those obtained by Pozzi and Lopo[1] and Merkin and Pop[12] respectively. Here, the magnetic parameter $M$ and conduction variation parameter $P$ are ignored (i.e. $M=0$ and $P=0$ ) and the Prandtl number $P r=$ 0.733 with $x^{\frac{1}{5}}=\xi$ is chosen. It is clearly seen that there is an excellent agreement among the present results with the solutions Pozzi and Lopo[1] and Merkin and Pop[12].

Table 1: Comparison of the present numerical results of skin friction coefficient $C_{f x}$ with Prandtl number $\operatorname{Pr}=$ $0.733, M=0$ and $P=0$, against $x$.

\begin{tabular}{|c|c|c|c|}
\hline \multicolumn{5}{|c|}{$C_{f x}$} \\
\hline$x^{\frac{1}{5}}=\xi$ & Pozzi and Lupo [1] & Merkin and Pop [12] & Present work \\
\hline 0.2 & & & \\
\hline 0.3 & 0.051 & 0.051 & 0.033 \\
\hline 0.4 & 0.105 & 0.105 & 0.033 \\
\hline 0.7 & 0.172 & 0.172 & 0.170 \\
\hline 0.8 & 0.430 & 0.430 & 0.423 \\
\hline 0.9 & 0.530 & 0.530 & 0.528 \\
\hline 1.0 & 0.635 & 0.635 & 0.633 \\
\hline 1.1 & 0.741 & 0.745 & 0.748 \\
\hline 1.2 & 0.829 & 0.859 & 0.857 \\
\hline & 0.817 & 0.972 & 0.972 \\
\hline
\end{tabular}


Table 2: Comparison of the present numerical results of surface temperature $\theta(x, 0)$ with Prandtl number $\operatorname{Pr}=0.733, M=0$ and $P=0$, against $x$.

\begin{tabular}{|c|c|c|c|}
\hline \multicolumn{4}{|c|}{$\theta(x, 0)$} \\
\hline$x^{\frac{1}{5}}=\xi$ & Pozzi and Lupo [1] & Merkin and Pop [12] & Present work \\
\hline 0.2 & 0.310 & 0.310 & 0.260 \\
\hline 0.3 & 0.413 & 0.413 & 0.260 \\
\hline 0.4 & 0.493 & 0.493 & 0.481 \\
\hline 0.7 & 0.651 & 0.651 & 0.651 \\
\hline 0.8 & 0.684 & 0.686 & 0.687 \\
\hline 0.9 & 0.708 & 0.715 & 0.716 \\
\hline 1.0 & 0.717 & 0.741 & 0.742 \\
\hline 1.1 & 0.699 & 0.762 & 0.763 \\
\hline 1.2 & 0.640 & 0.781 & 0.781 \\
\hline
\end{tabular}

\section{Conclusion}

The effects of the conduction variation on MHD free convective flow along a vertical flat plate have been studied in this paper. From the present investigation the following conclusions may be drawn:

The velocity within the boundary layer increases for decreasing values of $M, \operatorname{Pr}$ and $P$. There exists an inverse trend in velocity profiles for $P$.

The temperature within the boundary layer increases for increasing values of $M$ and decreases for increasing values of $P r$ and $P$.

The skin friction coefficient decreases for the increasing values of $M, P r$ and $P$. Mentionable effect is found in skin friction coefficient for $M, P r$ and $P$ which are all decreasing.

An increase in the values of $P r$ and $P$ leads to an increase in surface temperature. On the other hand, this decrease for increasing values of $M$. Remarkable effect is found in surface temperature for $\operatorname{Pr}$ and $P$ which are almost similar but inverse with $M$.

\section{REFERENCES}

[1] Pozzi, A. and Lupo, M., The coupling of conduction with laminar convection along a flat plate. Int. J. Heat Mass Transfer, Vol. 31, No. 9 (1988), 1807-1814.

[2] Pop, I. Lesnic, D. and Ingham, D. B. The conjugate mixed convection on a vertical surface in porous medium, Int. J. Heat transfer, Vol. 38, No. 8(1995), 1517-1525.

[3] Gebhart, B. Effect of dissipation on natural convection, J. Fluid Mechanics, Vol. 14, No. 2(1962), 225232.

[4] Takhar, H. S. and Soundalgekar, V. M. Dissipation effects on MHD free convection flow past a semiinfinite vertical plate, Applied Scientific Research, Vol. 36, No. 3 (1980), 163-171. 
[5] Khan, Z. I. Conjugate effect of conduction and convection with natural convection flow from a vertical flat plate and in an inclined square cavity, M. Phil thesis, Department of Mathematics, BUET, (2002).

[6] Mamun, A. A. Effects of conduction and convection on magnetohydrodynamic flow with and without dissipation from a vertical flat plate. M. Phil thesis, Department of Mathematics, BUET, (2005).

[7] Hossain, M. A. The viscous and Joule heating effects on MHD free convection flow with variable plate temperature, Int. J. Heat transfer Vol. 35, No. 12(1992), 3485-3487.

[8] Rahman, M.M, Mamun A.A, Azim M.A and Alim, M. A. Effects of temperature dependent thermal conductivity on magnetohydrodynamic (MHD) free convection flow along a vertical flat plate with heat conduction, Nonlinear Analysis: Modeling and Control, Vol.13, No. 4(2008), 513-524.

[9] Rahman, M. M and Alim, M. A. Numerical study of magnetohydrodynamic (MHD) free convective heat transfer flow along a vertical flat plate with temperature dependent thermal conductivity, Journal of Naval Architecture and Marine Engineering, JNAME, Vol.6, No.1(June, 2009), 16-29.

[10] Alim, M. A., Alam, Md. M., Mamun A.A. and Hossain, Md. B., Combined effect of viscous dissipation $\&$ joule heating on the coupling of conduction \& free convection along a vertical flat plate, Int. Commun. in Heat \& Mass Transfer, Vol. 35, No. 3(2008), 338-346.

[11] Alim, M. A., Alam, M. and Mamun, A. A., Joule heating effect on the coupling of conduction with MHD free convection flow from a vertical flat plate, Nonlinear Analysis: Modeling and Control, Vol.12, No. 3 (2007), 307-316.

[12] Merkin, J.H and Pop, I., Conjugate free convection on a vertical surface, Int. J. Heat Mass Transfer, Vol. 39 (1996), 1527-1534.

[13] Molla, M. Md, Rahman, A. and Rahman, T. L., Natural convection flow from an isothermal sphere with temperature dependent thermal conductivity. J. Architecture and Marine Engineering, Vol. 2 (2005), 53-64.

[14] Keller, H. B. Numerical methods in boundary layer theory, Annual Rev. Fluid Mech. Vol. 10 (1978), 417-433.

[15] Cebeci, T and Bradshaw, P. Physical and Computational Aspects of Convective Heat Transfer, Springer, New York, (1984).

[16] Chowdhury, M. K. and Islam, M.N. MHD Free Convection Flow of Visco-elastic Fluid past an Infinite Porous Plate, Int. J. Heat and Mass Transfer, Vol. 36, No. 5(2000), 43 9-447.

[17] Alam, Md. M., Alim, M. A. and Chowdhury, Md. M. K., Viscous dissipation effects on MHD natural convection flow over a sphere in the presence of heat generation, Nonlinear Analysis: Modeling and Control, Vol.12, No. 4(2007), 447-459.

[18] Islam, A.K.M. Safiqul, Alim M. A., Karim, Md. Rezaul and Rahman, ATM. M., Effects of Thermal Conductivity of Fluid on Free Convection Flow along a Vertical Flat Plate with Transverse Conduction, Journal of Mechanical Engineering, Vol. ME 44, No. 2(2014), 105-111. 